\title{
Exercício Aeróbio e Função Cardíaca de Murinos Expostos à Doxorrubicina: uma Metanálise
}

\author{
Aerobic Exercise and Cardiac Function of Murines Exposed to Doxorubicin: a Meta-Analysis
}

Mariana Inocêncio Matos, ${ }^{1,2}$ Ercole da Cruz Rubini, ${ }^{1,2,3}$ Frederico de Oliveira Meirelles, ${ }^{1,2,3(0)}$ Elirez Bezerra da Silva ${ }^{1,2}$ Universidade do Estado do Rio de Janeiro - Programa de Pós-Graduação em Ciências do Exercício e do Esporte, ${ }^{1}$ Rio de Janeiro, RJ - Brasil Universidade do Estado do Rio de Janeiro - Grupo de Pesquisa em Ciências do Exercício e da Saúde, ${ }^{2}$ Rio de Janeiro, RJ - Brasil

Universidade Estácio de Sá, ${ }^{3}$ Rio de Janeiro, RJ - Brasil

\section{Resumo}

Fundamento: A cardiotoxicidade pode ser uma consequência do tratamento com doxorrubicina (DOX).

Objetivos: Verificar o efeito do exercício aeróbio na prevenção da disfunção cardíaca de murinos expostos à DOX.

Método: Uma busca abrangente foi realizada em nove bases de dados, em dezembro de 2017. Estudos que avaliaram a função cardíaca de murinos expostos à DOX foram incluídos. O nível de significância adotado foi de 5\%.

Resultados: Na comparação entre 230 murinos submetidos a exercício aeróbio mais DOX e 222 controles (tratados somente com DOX), a fração de encurtamento mostrou uma melhora de $5,33 \%$ a favor do grupo experimental $(p=0,0001)$. A pressão desenvolvida no ventrículo esquerdo também mostrou um aumento de $24,84 \mathrm{mmHg}$ a favor do grupo de 153 murinos que realizaram exercício em comparação com o grupo controle de 166 murinos $(p=0,00001)$.

Conclusão: Estudos pré-clínicos incluídos nesta metanálise indicaram que o exercício é uma boa estratégia não farmacológica para preservar a função cardíaca pós-DOX. (Arq Bras Cardiol. 2020; 115(5):885-893)

Palavras-chave: Muridae; Exercício Físico; Antibacterianos; Doxorrubicina; Metanálise.

\section{Abstract}

Background: Cardiotoxicity may be a consequence of treatments with doxorubicin (DOX).

Objectives: To investigate the effect of aerobic exercise on the prevention of cardiac dysfunction in murines exposed to DOX.

Method: A comprehensive search was conducted in 9 databases in December 2017. Studies that evaluated the cardiac function of murines exposed to DOX were included. The significance level adopted was $5 \%$.

Results: In a comparison between 230 murines that underwent aerobic exercise plus DOX treatment and 222 control murines (DOX treatment only), fractional shortening showed an improvement of 5.33\% in favor of the experimental group ( $p=0.00001)$. Left ventricle developed pressure also showed an increase of $24.84 \mathrm{~mm} \mathrm{Hg}$ in favor of the group of 153 murines that performed exercise in comparison to the control group of 166 murines $(p=0.00001)$.

Conclusion: Preclinical studies included in this meta-analysis indicated that exercise is a good nonpharmacological strategy for preserving postDOX cardiac function. (Arq Bras Cardiol. 2020; 115(5):885-893)

Keywords: Muridae; Exercise; Anti-Bacterial Agents; Doxorubicin; Meta-Analysis.

Full texts in English - http://www.arquivosonline.com.br

Correspondência: Mariana Matos •

Universidade do Estado do Rio de Janeiro - Programa de Pós-Graduação em Ciências do Exercício e do Esporte - Rua São Francisco Xavier, 524,9 o andar. CEP 20550-900, Rio de Janeiro, RJ - Brasil

E-mail: mariana.i.matos@gmail.com

Artigo recebido em 31/05/2019, revisado em 04/08/2019, aceito em 10/09/2019

DOI: https://doi.org/10.36660/abc.20190260 


\section{Introdução}

A quimioterapia expõe um novo panorama na oncologia, no qual a sobrevida dos pacientes tem aumentado junto com a vulnerabilidade à cardiotoxicidade adquirida em tratamentos avançados. ${ }^{1}$ Os efeitos da toxicidade gerada pelos agentes antineoplásicos usados no tratamento podem se manifestar imediatamente, durante a administração ou até anos depois. ${ }^{2,3}$ Entre os órgãos afetados, o coração merece atenção especial, já que a insuficiência cardíaca, muitas vezes adquirida após o tratamento químico, tem prognóstico igual ou pior quando comparada a cânceres localizados no fígado, intestino, bexiga, próstata, mama e ovário. Assim, tais complicações podem interromper o tratamento e comprometer a probabilidade de cura. ${ }^{4}$

A doxorrubicina (DOX) é um agente quimioterápico eficiente na luta contra câncer de mama, tumores sólidos em crianças e linfomas agressivos. ${ }^{5}$ No entanto, estudos sugerem que a cardiotoxicidade da DOX promova uma diminuição na fração de ejeção do ventrículo esquerdo (FEVE). A incidência de cardiomiopatias em pacientes tratados anteriormente ou em tratamento atual com DOX é de $3 \%$ a $26 \%$, mas os dados sobre a prevalência ainda são escassos. ${ }^{6} \mathrm{~A}$ diminuição na FEVE pode iniciar com as primeiras doses de DOX e está relacionada à dose cumulativa. Doses abaixo de $550 \mathrm{mg} / \mathrm{m}^{2}$ podem reduzir a probabilidade de cardiomiopatias. ${ }^{2}$ Doses mais altas podem causar danos permanentes ao miocárdio, caracterizados por apoptose dos miócitos, que resulta em fibrose e consequente perda de função cardíaca. ${ }^{3}$

O estresse oxidativo potencializado pela DOX parece dar início a uma série de processos bioquímicos nas fibras do músculo cardíaco, que resultam em danos ao retículo sarcoplasmático e à mitocôndria, modificações funcionais e estruturais das miofibrilas e modificação do acoplamento de excitação-contração e do fluxo de cálcio. Essas alterações levam à apoptose e, por fim, à perda da capacidade de regeneração do músculo cardíaco. ${ }^{7}$

Alguns dos benefícios do exercício são a melhora do funcionamento do sistema imunológico, a redução de atividade inflamatória e a atenuação dos efeitos metabólicos da imobilidade e da quimioterapia, que tornam o exercício uma excelente ferramenta não farmacológica para reduzir os efeitos tóxicos da DOX e ajudar a melhorar a qualidade de vida dos pacientes submetidos a tratamento..$^{8,9} \mathrm{O}$ potencial cardioprotetor do exercício contra a cardiotoxicidade parece estar ligado a diversos mecanismos moleculares, como produção antioxidante aumentada, regulação de sinalização pró-apoptótica, limitação da renovação de miócitos, modulação da atividade cardíaca da proteína quinase ativada por monofostato de adenosina (AMPK), regulação negativa da autofagia cardíaca, redução do acúmulo cardíaco de DOX, entre outros. ${ }^{10-12}$

Estudos sobre exercício e disfunção cardíaca causada por cardiotoxicidade de DOX em humanos ainda são limitados, mas há um número razoável de estudos pré-clínicos na literatura. Assim, esta metanálise foi realizada com estudos pré-clínicos e, até onde sabemos, é a primeira sobre o assunto. O objetivo foi verificar os efeitos de exercício aeróbio na função cardíaca de murinos expostos a DOX.

\section{Métodos}

\section{Critérios de Inclusão}

Foram incluídos ensaios clínicos randomizados (ECRs) com modelos de murinos que realizaram exercício aeróbio antes, durante e após a exposição à DOX, comparados com o grupo controle. A função cardíaca deveria ter sido medida pela fração de encurtamento $(\triangle \mathrm{D} \%)$ e pela pressão desenvolvida no ventrículo esquerdo (PDVE).

\section{Critérios de Exclusão}

Foram excluídos estudos com delineamentos diferentes dos ECRs, estudos em que o grupo de exercício experimental usou medicamento concomitante, estudos com humanos ou estudos sem média e desvio padrão dos resultados relativos à $\triangle \mathrm{D} \%$ e PDVE.

\section{Busca}

A busca foi conduzida em dezembro de 2017 nas bases de dados digitais MEDLINE, LILACS, CENTRAL Cochrane, PEDro, CINAHL, ScienceDirect, SPORTDiscus, Scopus e Web of Science. Os descritores cardiotoxicidade, câncer, doxorrubicina, exercício e todos os sinônimos presentes nas bases de dados Medical Subject Headings e Descriptors in Health Sciences foram utilizados na busca.

\section{Dados Extraídos dos Estudos}

Os tipos de exercícios, os protocolos de treinamento, as dosagens de infusões de DOX, as formas de aplicação, os períodos de exposição ao fármaco, os resultados da $\Delta \mathrm{D} \%$ e da PDVE $(\mathrm{mmHg})$ e o tamanho das amostras utilizadas foram extraídos dos estudos selecionados para revisão.

Para a análise da função cardíaca in vivo, foi considerada a $\triangle \mathrm{D} \%$ do ventrículo esquerdo medida por ecocardiografia e Doppler. A $\Delta \mathrm{D} \%$ é um dos principais parâmetros a serem monitorados em pacientes expostos a terapias cardiotóxicas, já que é uma medida indicativa da função sistólica do ventrículo esquerdo. ${ }^{13,14}$

Uma avaliação da PDVE através de um transdutor posicionado no ventrículo esquerdo (VE), baseada no modelo de isolamento cardíaco de Langendorff, é comum nesses estudos. Assim, a análise ex vivo também foi considerada para verificar a capacidade de contração do VE. ${ }^{15}$

\section{Análise da Qualidade Metodológica}

Um avaliador independente analisou o risco de viés em cada estudo incluído na metanálise através da ferramenta da Cochrane Collaboration para determinar o risco de viés em ensaios clínicos randomizados (2005-2007), disponível para download em http://www.cochrane-handbook.org. Seguindo as recomendações do Cochrane Handbook for Systematic Reviews of Interventions, ${ }^{16}$ foi usada a abordagem GRADE através do GRADEproGDT, disponível em https://gradepro. org/, para analisar o nível de evidências para cada desfecho $(\triangle \mathrm{D} \%$ e $P D V E)$. 


\section{Análise Estatística}

O programa Review Manager v. 5.3, com desfecho contínuo, método estatístico de variância inversa, análise por modelo de efeitos aleatórios, medida de efeito por diferença da média e intervalo de confiança (IC) de 95\%, foi usado para os estudos, para a metanálise e para ordenar os estudos por peso. O nível de significância adotado foi de $5 \%$.

\section{Resultados}

As etapas realizadas na busca dos manuscritos estão descritas no diagrama da Figura 1. Entre os nove estudos selecionados para análise, sete apresentaram resultados de $\triangle \mathrm{D} \%$ e quatro apresentaram resultados de PDVE. Apenas dois estudos apresentaram as duas variáveis analisadas.
O estudo de Hydock et al. ${ }^{19}$ (2012) testou dois protocolos de injeção de DOX diferentes. Assim, foi dividido em duas análises, nomeadas "a" e "b". Para verificar a influência do hormônio feminino na cardiotoxicidade induzida por DOX, o estudo de Calvé et al., ${ }^{20}$ foi dividido em "a", com ratos em condições normais, e "b", com ratos ovariectomizados. Em uma análise com dois protocolos de exercício diferentes, o estudo de Jensen et al. (2013) 22 foi dividido em "a", em que os ratos foram submetidos ao protocolo de esteira progressiva, e " $b$ ", em que o grupo tinha acesso livre à roda de corrida. Para entender melhor os resultados e as comparações, Lien et al., ${ }^{24}$ (2015) conduziram quatro estudos ("a", "b", "c" e "d"), de acordo com o número de grupos ativos (Tabelas 1 e 2).

Os resultados da $\triangle \mathrm{D} \%$, da PDVE e da $\mathrm{I}^{2}$ desta metanálise são mostrados nas Figuras 2 e 3.

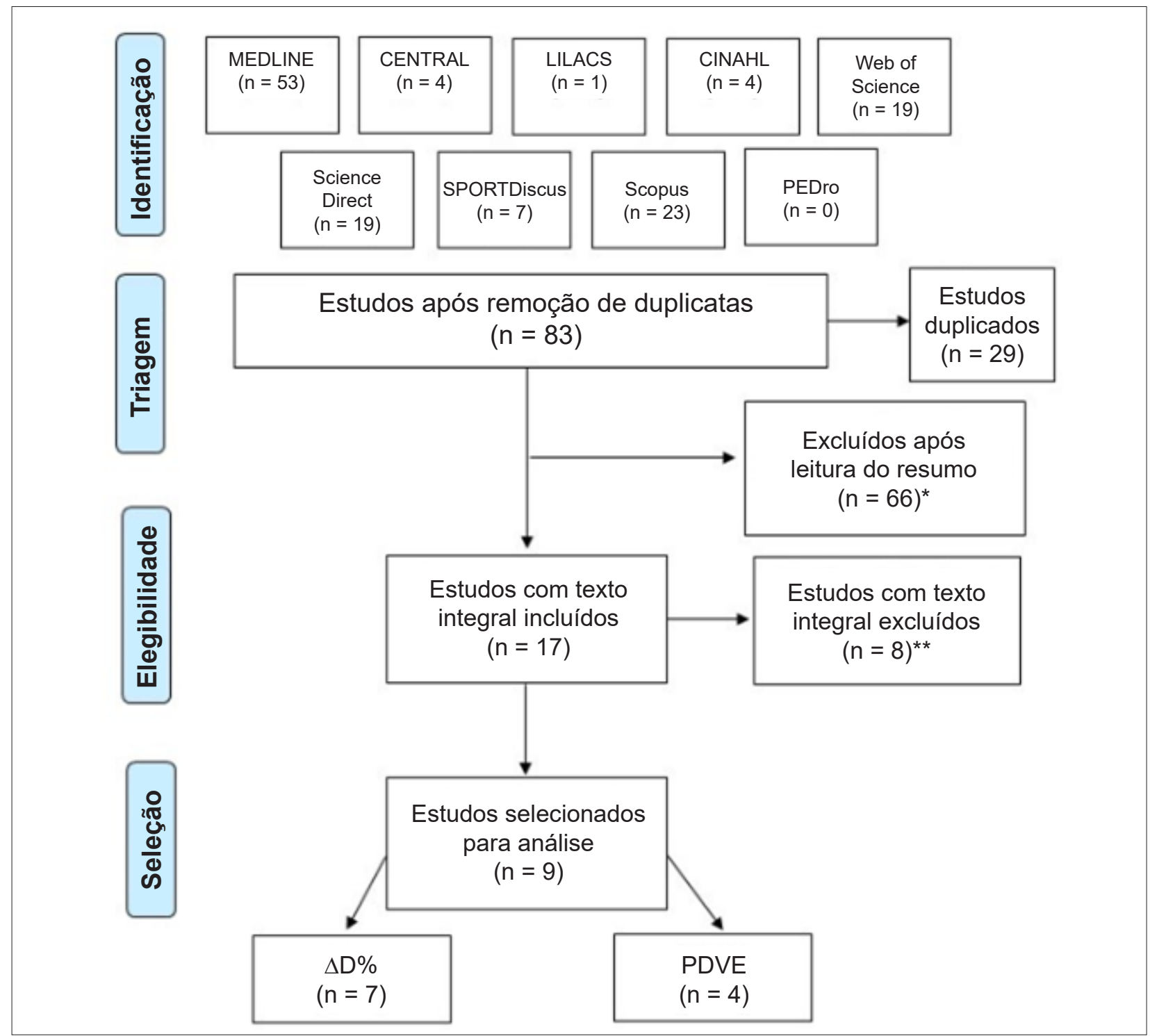

Figura 1 - Diagrama de fluxo dos estudos (Prisma, 2009)18. *Estudos excluídos por não atenderem aos critérios de exclusão. **Estudos excluídos pelo uso concomitante de medicamento no grupo experimental, inclusão de seres humanos e/ou ausência de média e desvio padrão para a resposta da função cardíaca. $\Delta D \%$ : fração de encurtamento; PDVE: pressão desenvolvida no ventrículo esquerdo. 
Tabela 1 - Resumo dos estudos selecionados para a variável fração de encurtamento $(\Delta D \%)$ do ventrículo esquerdo.

\begin{tabular}{|c|c|c|c|c|c|c|c|}
\hline \multirow{3}{*}{ Autor (ano) } & \multirow{3}{*}{$\begin{array}{l}\text { Tipo de } \\
\text { exercício }\end{array}$} & \multirow{3}{*}{$\begin{array}{l}\text { Protocolo de } \\
\text { intervenção }\end{array}$} & \multirow{3}{*}{ Injeção de DOX } & \multicolumn{4}{|c|}{ Fração de encurtamento do ventrículo esquerdo ( $\Delta \mathrm{D} \%)$} \\
\hline & & & & \multicolumn{2}{|c|}{ Controle + DOX } & \multicolumn{2}{|c|}{ Exercício aeróbio + DOX } \\
\hline & & & & $\mathrm{n}$ & $\overline{\mathbf{X}}_{D P}$ & $\mathrm{n}$ & $\overline{\mathbf{X}}_{\mathrm{DP}}$ \\
\hline $\begin{array}{l}\text { Hayward et al. } \\
(2012)^{18}\end{array}$ & $\begin{array}{l}\text { Aeróbio } \\
\text { Roda de } \\
\text { corrida }\end{array}$ & $\begin{array}{c}\text { Acesso livre } 24 \\
\text { h/dia Total: } 10 \\
\text { semanas }\end{array}$ & $\begin{array}{c}2 \mathrm{mg} / \mathrm{kg} \text { por } 7 \text { dias } \\
\text { Total = } 14 \mathrm{mg} / \mathrm{kg} \\
\text { durante o exercício }\end{array}$ & 15 & $52 \pm 38$ & 17 & $61 \pm 29$ \\
\hline $\begin{array}{l}\text { Hydock et al. } \\
(2012) \text { (a) }^{19}\end{array}$ & $\begin{array}{l}\text { Aeróbio } \\
\text { Roda de } \\
\text { corrida }\end{array}$ & $\begin{array}{l}\text { Acesso livre } 24 \\
\text { h/dia Total: } 10 \\
\text { semanas }\end{array}$ & $\begin{array}{c}1 \mathrm{mg} / \mathrm{kg} \text { por } 15 \mathrm{dias} \\
\text { Total = } 15 \mathrm{mg} / \mathrm{kg} \\
\text { durante o exercício }\end{array}$ & 15 & $45 \pm 3$ & 9 & $46 \pm 4$ \\
\hline $\begin{array}{l}\text { Hydock et al. } \\
(2012)(b)^{19}\end{array}$ & $\begin{array}{l}\text { Aeróbio } \\
\text { Roda de } \\
\text { corrida }\end{array}$ & $\begin{array}{l}\text { Acesso livre } 24 \\
\text { h/dia Total: } 10 \\
\text { semanas }\end{array}$ & $\begin{array}{c}2,5 \mathrm{mg} / \mathrm{kg} \\
\text { semanalmente por } 6 \\
\text { semanas } \\
\text { Total }=15 \mathrm{mg} / \mathrm{kg} \\
\text { durante o exercício }\end{array}$ & 10 & $52 \pm 5$ & 10 & $61 \pm 4$ \\
\hline $\begin{array}{l}\text { Calvé et al. } \\
(2012)(a)^{20}\end{array}$ & $\begin{array}{l}\text { Aeróbio } \\
\text { Nado }\end{array}$ & $\begin{array}{c}1 \mathrm{~h} / \mathrm{dia} \\
\text { Total: } 4 \text { semanas }\end{array}$ & $\begin{array}{c}3 \mathrm{mg} / \mathrm{kg} \text { no } 26^{\circ} \text { dia } \\
\text { de vida } \\
\text { pré-exercício }\end{array}$ & 8 & $53,1 \pm 3,8$ & 8 & $49,5 \pm 2,2$ \\
\hline $\begin{array}{l}\text { Calvé et al. } \\
(2012)(b)^{20}\end{array}$ & $\begin{array}{l}\text { Aeróbio } \\
\text { Nado }\end{array}$ & $\begin{array}{c}1 \mathrm{~h} / \mathrm{dia} \\
\text { Total: } 4 \text { semanas }\end{array}$ & $\begin{array}{c}3 \mathrm{mg} / \mathrm{kg} \text { no } 26^{\circ} \text { dia } \\
\text { de vida } \\
\text { pré-exercício }\end{array}$ & 8 & $47 \pm 2,1$ & 8 & $51,6 \pm 1,7$ \\
\hline $\begin{array}{l}\text { Dolisnky et al. } \\
(2013)^{21}\end{array}$ & $\begin{array}{l}\text { Aeróbio } \\
\text { Esteira }\end{array}$ & $\begin{array}{c}10-18 \mathrm{~m} / \mathrm{min} \\
5 \mathrm{dias} / \mathrm{semana} \\
\text { Total: } 8 \text { semanas }\end{array}$ & $\begin{array}{c}8 \mathrm{mg} / \mathrm{kg} \text { por semana } \\
\text { por } 4 \text { semanas } \\
\text { Total = } 32 \mathrm{mg} / \mathrm{kg} \\
\text { pré-exercício }\end{array}$ & 8 & $23,8 \pm 1,0$ & 8 & $28,0 \pm 0,7$ \\
\hline $\begin{array}{l}\text { Jensen et al. } \\
(2013)(a)^{22}\end{array}$ & $\begin{array}{l}\text { Aeróbio } \\
\text { Esteira }\end{array}$ & $\begin{array}{c}13-30 \mathrm{~min} / \mathrm{m} \\
5^{0}-18^{\circ} \\
20-60 \mathrm{~min} / \mathrm{dia} \\
5 \text { dias } / \mathrm{semana} \\
\text { Total: } 10 \text { semanas }\end{array}$ & $\begin{array}{l}10 \mathrm{mg} / \mathrm{kg} \\
\text { dose única pós- } \\
\text { exercício }\end{array}$ & 8 & $50,47 \pm 2,77$ & 4 & $61,60 \pm 7,28$ \\
\hline $\begin{array}{l}\text { Jensen et al. } \\
(2013)(b)^{22}\end{array}$ & $\begin{array}{l}\text { Aeróbio } \\
\text { Roda de } \\
\text { corrida }\end{array}$ & $\begin{array}{c}\text { Acesso livre } 24 \\
\text { h/dia Total: } 10 \\
\text { semanas }\end{array}$ & $\begin{array}{c}10 \mathrm{mg} / \mathrm{kg} \\
\text { dose única pós- } \\
\text { exercício }\end{array}$ & 8 & $50,47 \pm 2,77$ & 7 & $58,3 \pm 4,33$ \\
\hline $\begin{array}{l}\text { Parry et al. } \\
(2015)^{23}\end{array}$ & $\begin{array}{l}\text { Aeróbio } \\
\text { Roda de } \\
\text { corrida }\end{array}$ & $\begin{array}{l}\text { Acesso livre } 24 \\
\text { h/dia Total: } 11 \\
\text { semanas }\end{array}$ & $\begin{array}{c}12 \mathrm{mg} / \mathrm{kg} \\
\text { dose única pós- } \\
\text { exercício }\end{array}$ & 6 & $59 \pm 6 \dagger$ & 4 & $63 \pm 4 \dagger$ \\
\hline $\begin{array}{l}\text { Lien et al. } \\
(2015)(a)^{24}\end{array}$ & $\begin{array}{l}\text { Aeróbio } \\
\text { Esteira }\end{array}$ & $\begin{array}{l}18-24 \mathrm{~m} / \mathrm{min} \\
\text { Total: } 5 \text { dias }\end{array}$ & $\begin{array}{c}10 \mathrm{mg} / \mathrm{kg} \\
\text { dose única pós- } \\
\text { exercício }\end{array}$ & 10 & $48 \pm 4$ & 10 & $56 \pm 4$ \\
\hline $\begin{array}{l}\text { Lien et al. } \\
(2015)(b)^{24}\end{array}$ & $\begin{array}{l}\text { Aeróbio } \\
\text { Roda de } \\
\text { corrida }\end{array}$ & $\begin{array}{c}\text { Acesso livre } 24 \text { h/ } \\
\text { dia Total: } 5 \text { dias }\end{array}$ & $\begin{array}{c}10 \mathrm{mg} / \mathrm{kg} \\
\text { dose única pós- } \\
\text { exercício }\end{array}$ & 10 & $48 \pm 4$ & 10 & $51 \pm 5$ \\
\hline $\begin{array}{l}\text { Lien et al. } \\
(2015)(c)^{24}\end{array}$ & $\begin{array}{l}\text { Aeróbio } \\
\text { Esteira }\end{array}$ & $\begin{array}{l}18-24 \mathrm{~m} / \mathrm{min} \\
\text { Total: } 5 \text { dias }\end{array}$ & $\begin{array}{c}15 \mathrm{mg} / \mathrm{kg} \\
\text { dose única pós- } \\
\text { exercício }\end{array}$ & 13 & $39 \pm 6$ & 13 & $48 \pm 5$ \\
\hline $\begin{array}{l}\text { Lien et al. } \\
(2015)(d)^{24}\end{array}$ & $\begin{array}{l}\text { Aeróbio } \\
\text { Roda de } \\
\text { corrida }\end{array}$ & $\begin{array}{c}\text { Acesso livre } 24 \text { h/ } \\
\text { dia Total: } 5 \text { dias }\end{array}$ & $\begin{array}{c}15 \mathrm{mg} / \mathrm{kg} \\
\text { dose única pós- } \\
\text { exercício }\end{array}$ & 13 & $39 \pm 6$ & 12 & $45 \pm 3$ \\
\hline
\end{tabular}

DOX: doxorrubicina; x: média; DP: desvio padrão; t: medidas feitas no $5^{\circ}$ dia após a injeção de DOX; (a), (b), (c), (d) são subdivisões dos estudos de Hydock et al. ${ }^{19}$ (2012), Calvé et al. ${ }^{20}$ (2012), Jensen et al. ${ }^{22}$ (2013) e Lien et al. ${ }^{24}$ (2015). 


\begin{tabular}{|c|c|c|c|c|c|c|c|}
\hline \multirow{3}{*}{ Autor (ano) } & \multirow{3}{*}{$\begin{array}{l}\text { Tipo de } \\
\text { exercício }\end{array}$} & \multirow{3}{*}{$\begin{array}{l}\text { Protocolo de } \\
\text { intervenção }\end{array}$} & \multirow{3}{*}{ Injeção de DOX } & \multicolumn{4}{|c|}{ Pressão desenvolvida no ventrículo esquerdo (PDVE), mmHg } \\
\hline & & & & \multicolumn{2}{|c|}{ Controle + DOX } & \multicolumn{2}{|c|}{ Exercício aeróbio + DOX } \\
\hline & & & & $\mathrm{n}$ & $\overline{\mathbf{X}_{D P}}$ & $\mathrm{n}$ & $\overline{\mathbf{X}_{D P}}$ \\
\hline $\begin{array}{l}\text { Chicco et al. } \\
(2005)^{25}\end{array}$ & $\begin{array}{c}\text { Aeróbio } \\
\text { Roda de corrida }\end{array}$ & $\begin{array}{c}\text { Acesso livre } 24 \\
\text { h/dia Total: } 8 \\
\text { semanas }\end{array}$ & 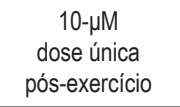 & 7 & $30,5 \pm 1,4$ & 7 & $50,1 \pm 7,7$ \\
\hline $\begin{array}{l}\text { Chicco et al. } \\
(2006)^{26}\end{array}$ & $\begin{array}{l}\text { Aeróbio } \\
\text { Esteira }\end{array}$ & $\begin{array}{c}15-27 \mathrm{~m} / \mathrm{min} \\
0^{0}-5^{\circ} \\
20-60 \mathrm{~min} / \mathrm{dia} \\
5 \text { dias } / \mathrm{semana} \\
\text { Total: } 12 \text { semanas }\end{array}$ & $\begin{array}{c}15 \mathrm{mg} / \mathrm{kg} \\
\text { dose única } \\
\text { pós-exercício }\end{array}$ & 15 & $46 \pm 9$ & 15 & $84 \pm 7$ \\
\hline $\begin{array}{l}\text { Hayward et al. } \\
(2012)^{18}\end{array}$ & $\begin{array}{c}\text { Aeróbio } \\
\text { Roda de corrida }\end{array}$ & $\begin{array}{l}\text { Acesso livre } 24 \\
\text { h/dia Total: } 10 \\
\text { semanas }\end{array}$ & $\begin{array}{l}2 \mathrm{mg} / \mathrm{kg} \text { por } 7 \\
\text { dias } \\
\text { Total }=14 \mathrm{mg} / \\
\mathrm{kg} \text { durante o } \\
\text { exercício }\end{array}$ & 22 & $91 \pm 15 \dagger$ & 22 & $121 \pm 12 \dagger$ \\
\hline $\begin{array}{l}\text { Jensen et al. } \\
(2013)(a)^{22}\end{array}$ & $\begin{array}{l}\text { Aeróbio } \\
\text { Esteira }\end{array}$ & $\begin{array}{c}13-30 \mathrm{~min} / \mathrm{m} \\
5^{\circ}-18 \mathrm{~m} \\
20-60 \mathrm{~min} / \mathrm{dia} \\
5 \text { dias/semana } \\
\text { Total: } 10 \text { semanas }\end{array}$ & $\begin{array}{c}10 \mathrm{mg} / \mathrm{kg} \\
\text { dose única } \\
\text { pós-exercício }\end{array}$ & 14 & $70 \pm 3+\dagger$ & 10 & $93 \pm 3+\dagger$ \\
\hline $\begin{array}{l}\text { Jensen et al. } \\
(2013)_{(b)^{22}}\end{array}$ & $\begin{array}{c}\text { Aeróbio } \\
\text { Roda de corrida }\end{array}$ & $\begin{array}{l}\text { Acesso livre } 24 \\
\text { h/dia Total: } 10 \\
\text { semanas }\end{array}$ & $\begin{array}{c}10 \mathrm{mg} / \mathrm{kg} \\
\text { dose única } \\
\text { pós-exercício }\end{array}$ & 14 & $70 \pm 3+\dagger$ & 10 & $89 \pm 2 \dagger \dagger$ \\
\hline
\end{tabular}

DOX: doxorrubicina; x: média; DP: desvio padrão; (a) e (b) são subdivisões de Jensen et al.22 (2013); †: medida feita com 300 batimentos por minuto; †t: medida feita no $9^{\circ}$ dia após a injeção de DOX.

O risco de viés dos estudos e o nível de evidência da metanálise são mostrados nas Tabelas 3 e 4, respectivamente.

\section{Discussão}

No presente estudo, a disfunção cardíaca resultante do uso de DOX foi avaliada através de $\triangle \mathrm{D} \%$ e PDVE $(\mathrm{mmHg})$, que estão relacionadas à função sistólica do ventrículo esquerdo. A metanálise dos resultados de 230 murinos submetidos a exercício aeróbio mais DOX e de 222 controles (tratados somente com DOX) mostrou uma melhora na $\Delta \mathrm{D} \%$ de 5,33 $(p=0,00001)$ nos murinos que realizaram exercício aeróbio (Figura 2). Da mesma forma, a metanálise dos resultados de 153 murinos submetidos a exercício aeróbio mais DOX e dos 166 controles (tratados somente com DOX) mostrou um aumento na PDVE de 24,84 mmHg ( $p=0,00001$ ) nos murinos que realizaram exercício aeróbio (Figura 3). Resumidamente, a prática de exercício aeróbio contribuiu para a melhora da função sistólica, ou seja, para a diminuição da disfunção cardíaca causada pelo uso de DOX.

A toxicidade das antraciclinas causa disfunção grave em todos os tecidos musculares. No entanto, as células do músculo cardíaco parecem acumular maiores quantidades de DOX do que as células dos músculos liso e esquelético. ${ }^{27}$ Por isso, a detecção precoce dos fatores de risco cardiovascular, a monitorização cuidadosa dos parâmetros das funções diastólica e sistólica do ventrículo esquerdo e a medição da FEVE e da pressão de enchimento do ventrículo esquerdo devem ser realizadas periodicamente em pacientes submetidos a quimioterapia para evitar a perda permanente de função do músculo cardíaco devido à cardiotoxicidade. ${ }^{28}$

O exercício aeróbio parece promover a liberação dos antioxidantes, protegendo, assim, a fibra cardíaca de danos causados pela liberação excessiva de espécies reativas de oxigênio após a exposição à DOX. ${ }^{29-32}$ Esse efeito oxidativo antiestresse é percebido quando o exercício é realizado sistematicamente, antes ou depois da exposição ao fármaco. ${ }^{33}$ Embora as células tenham um sistema antioxidante endógeno, os cardiomiócitos têm uma capacidade de ativação desse sistema muito baixa quando comparados com células de outros tecidos. ${ }^{34,35}$ Assim, o exercício aeróbio tem se mostrado uma boa estratégia não farmacológica para combater a cardiotoxicidade. ${ }^{36}$

Em seres humanos, a prática regular de exercício aeróbio de três a quatro vezes por semana por 40 minutos, com atividades de intensidade moderada a extrema, parece ter efeito direto na prevenção de doenças cardiovasculares independentemente de outros fatores de riso, além de contribuir para taxas mais baixas de mortalidade cardíaca. ${ }^{37,38}$ Essa frequência de exercícios também afeta a produção de radicais livres, o que protege pacientes treinados dos efeitos crônicos gerados pelo estresse oxidativo de atividades físicas diárias. ${ }^{39}$ 


\begin{tabular}{|c|c|c|c|c|c|c|c|c|c|c|c|}
\hline \multirow[b]{2}{*}{$\begin{array}{l}\text { Estudo ou } \\
\text { subgrupo }\end{array}$} & \multicolumn{3}{|c|}{ Exercício aeróbio } & \multicolumn{3}{|c|}{ Controle } & \multirow[b]{2}{*}{ Peso } & \multirow{2}{*}{$\begin{array}{l}\text { Diferença média } \\
\text { VI, Aleatório, } \\
\text { IC95\% }\end{array}$} & & \multirow{2}{*}{\multicolumn{2}{|c|}{$\begin{array}{c}\text { Diferença média } \\
\text { VI, Aleatório, IC95\% }\end{array}$}} \\
\hline & Média & \multirow{2}{*}{29} & \multirow{2}{*}{$\begin{array}{c}\text { Total } \\
17\end{array}$} & \multirow{2}{*}{$\begin{array}{c}\text { Média } \\
52\end{array}$} & \multirow{2}{*}{\begin{tabular}{|l} 
DP \\
38
\end{tabular}} & \multirow{2}{*}{$\begin{array}{c}\text { Total } \\
15\end{array}$} & & & & & \\
\hline Hayward et al. (2012) & 61 & & & & & & \multirow{2}{*}{$\begin{array}{l}0,6 \% \\
6,8 \% \\
\end{array}$} & $9,00[-14,66,32,66]$ & & & \\
\hline Lien et al. (2015) c & 48 & 5 & 13 & 39 & 6 & 13 & & $9,00[4,75,13,25]$ & & & \\
\hline $\begin{array}{l}\text { Hydock et al. } \\
\text { (2012) b }\end{array}$ & 61 & 4 & 10 & 52 & 5 & 10 & $7,1 \%$ & $9,00[5,03,12,97]$ & & & $\vec{\square}$ \\
\hline Lien et al. (2015) b & 51 & 5 & 10 & 48 & 4 & 10 & $7,1 \%$ & $3,00[-0,97,6,97]$ & & & $\rightarrow$ \\
\hline Lien et al. (2015) d & 45 & 3 & 12 & 39 & 6 & 13 & $7,5 \%$ & $6,00[2,32,9,68]$ & & & $\longrightarrow$ \\
\hline Lien et al. (2015) a & 56 & 4 & 10 & 48 & 4 & 10 & $7,6 \%$ & $8,00[4,49,11,51]$ & & & \\
\hline Calvé et al. (2012) a & 49,5 & 2,2 & 8 & 53,1 & 3,8 & 8 & $8,2 \%$ & $-3,60[-6,64,-0,56]$ & & & - \\
\hline $\begin{array}{l}\text { Hydock et al. } \\
\text { (2012) a }\end{array}$ & 46 & 4 & 9 & 45 & 3 & 15 & $8,2 \%$ & $1,00[-2,02,4,02]$ & & & - \\
\hline Parry et al. (2015) & 63 & 4 & 36 & 59 & 6 & 36 & $8,9 \%$ & $4,00[1,64,6,36]$ & & & - \\
\hline Jensen et al. (2013) a & 61,6 & 7,28 & 47 & 50,47 & 2,77 & 38 & $9,0 \%$ & $11,13[8,87,13,39]$ & & & - \\
\hline Calvé et al. (2012) b & 51,6 & 1,7 & 8 & 47 & 2,1 & 8 & $9,3 \%$ & $4,60[2,73,6,47]$ & & & $\Leftrightarrow$ \\
\hline Jensen et al. (2013) b & 58,3 & 4,33 & 42 & 50,47 & 2,77 & 38 & $9,6 \%$ & $7,83[6,25,9,41]$ & & & \\
\hline Dolinsky et al. (2013) & 28 & 0,7 & 8 & 23,8 & 1 & 8 & $10,0 \%$ & $4,20[3,35,5,05]$ & -20 & $\begin{array}{c}-10 \\
\text { Control }\end{array}$ & ${ }_{\text {Aerobic exercise }}^{10} 20$ \\
\hline Total (IC 95\%) & & & 230 & & & 222 & $100,00 \%$ & $5,33[3,40,7,26]$ & & & \\
\hline $\begin{array}{l}\text { Heterogeneidade: } T \\
\text { Teste para efeito ge }\end{array}$ & $\begin{array}{l}=9,46 \\
z=5,4\end{array}$ & $\begin{array}{l}h^{2}=9 \\
P<0,\end{array}$ & $\begin{array}{l}76 ; d f= \\
0001)\end{array}$ & $P<0$, & 1); $; 1$ & & & & & & \\
\hline
\end{tabular}

Figura 2 - Gráfico de floresta dos estudos com murinos expostos à $D O X$ que compararam a $\Delta D \%$ de um grupo que realizou exercício aeróbio à de um grupo controle sedentário. DP: desvio padrão; DOX: doxorrubicina; $\triangle D \%$ : fração de encurtamento; VI: variância inversa; IC: intervalo de confiança.

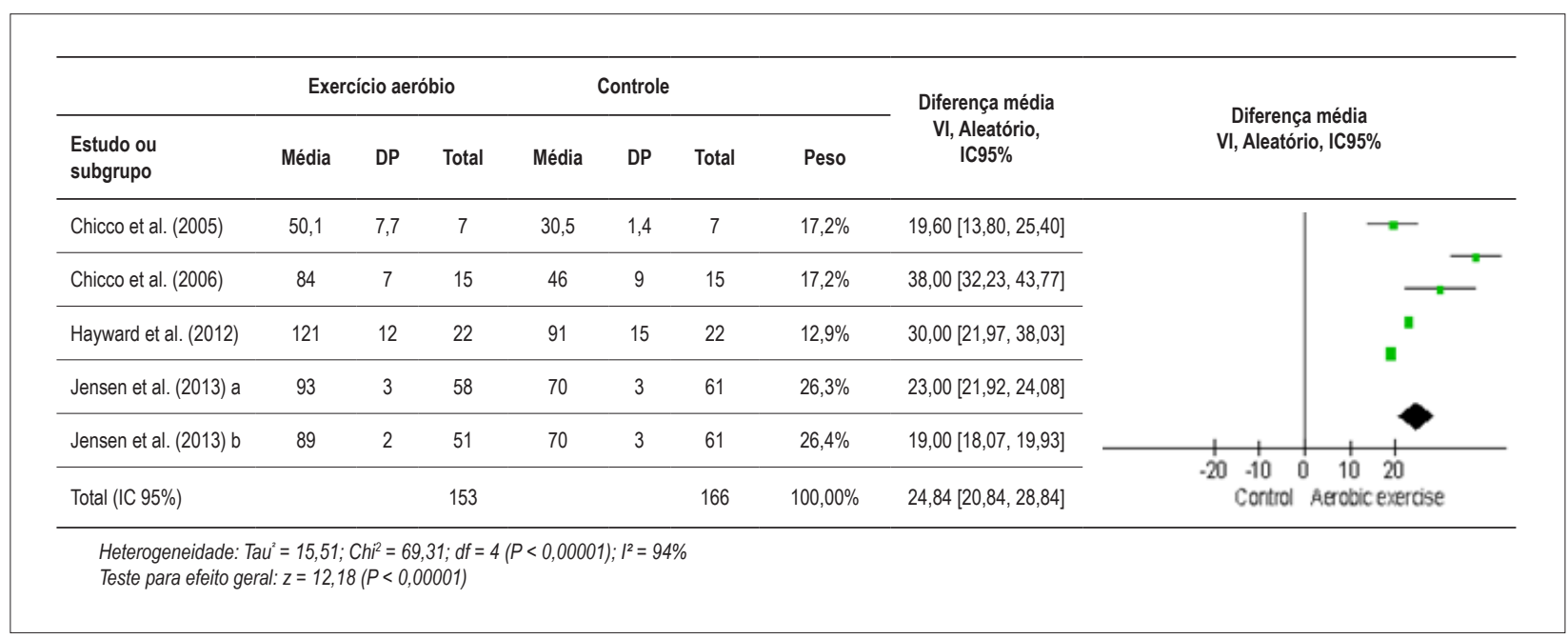

Figura 3 - Gráfico de floresta dos estudos com murinos que compararam a PDVE de um grupo que realizou exercício aeróbio à de um grupo controle sedentário. DP: desvio padrão; PDVE: pressão desenvolvida no ventrículo esquerdo; VI: variância inversa; IC: intervalo de confiança.

Um estudo metaepidemiológico mostrou que a intervenção com exercício aeróbio tem efeito similar ao de fármacos como betabloqueadores e inibidores de enzima de conversão da angiotensina nas taxas de mortalidade e prevenção secundária em pacientes com doenças coronárias, reabilitação de acidente vascular cerebral e tratamento de insuficiência cardíaca. ${ }^{40}$ Assim, é importante considerar o tratamento não farmacológico com exercícios para pacientes expostos a intervenções que acentuam o risco de doenças cardiovasculares, como a quimioterapia.
Outro aspecto positivo do exercício está relacionado à fadiga, a qual, além de ser um sintoma primário de muitos eventos cardíacos, é comum em pacientes expostos a quimioterapia. Puetz, Beasman e O'Connor ${ }^{41}$ concluíram, em uma metanálise, que programas de exercício para reabilitação cardíaca são associados à percepção de aumento de energia e diminuição de fadiga. ${ }^{41}$

Os resultados encontrados nesta metanálise a favor do grupo submetido a exercícios aeróbios mais DOX são fortalecidos pelos achados de revisões sistemáticas prévias 


\section{Artigo Original}

\begin{tabular}{lcccccccc}
\hline Tabela 3 - Ferramenta para avaliação de risco de viés da Cochrane Collaboration & & & & \\
\hline Autor (ano) & Randomização & $\begin{array}{c}\text { Ocultação da } \\
\text { randomização }\end{array}$ & $\begin{array}{c}\text { Cegamento dos } \\
\text { participantes }\end{array}$ & $\begin{array}{c}\text { Cegamento dos } \\
\text { avaliadores }\end{array}$ & $\begin{array}{c}\text { Desfechos } \\
\text { incompletos }\end{array}$ & $\begin{array}{c}\text { Relato seletivo } \\
\text { de desfechos }\end{array}$ & $\begin{array}{c}\text { Outras fontes } \\
\text { de viés }\end{array}$ & $\begin{array}{c}\text { Risco de } \\
\text { viés }\end{array}$ \\
\hline Chicco et al. $(2005)^{25}$ & Baixo & Baixo & Baixo & Baixo & Baixo & Baixo & Baixo & Baixo \\
\hline Chicco et al. $(2006)^{26}$ & Baixo & Baixo & Baixo & Baixo & Baixo & Baixo & Baixo & Baixo \\
\hline Hayward et al. $(2012)$ & Baixo & Baixo & Baixo & Baixo & Baixo & Baixo & Baixo & Baixo \\
\hline Hydock et al. $(2012)^{18}$ & Baixo & Baixo & Baixo & Baixo & Baixo & Baixo & Baixo & Baixo \\
\hline Calvé et al. $(2012)^{20}$ & Baixo & Baixo & Baixo & Baixo & Baixo & Baixo & Baixo & Baixo \\
\hline Jensen et al. $(2013)^{22}$ & Baixo & Baixo & Baixo & Baixo & Baixo & Baixo & Baixo & Baixo \\
\hline $\begin{array}{l}\text { Dolionsky et al. } \\
(2013)^{21}\end{array}$ & Baixo & Baixo & Baixo & Baixo & Baixo & Baixo & Baixo & Baixo \\
\hline Parry et al. $(2015)^{23}$ & Baixo & Baixo & Baixo & Baixo & Baixo & Baixo & Baixo & Baixo \\
\hline Lien et al. $(2015)^{24}$ & Baixo & Baixo & Baixo & Baixo & Baixo & Baixo & Baixo & Baixo \\
\hline
\end{tabular}

* Os itens referentes à randomização e ao cegamento da amostra foram considerados como tendo baixo risco de viés mesmo quando não estava explícito no ensaio clínico randomizado, já que estudos com modelos de murinos neutralizam esses vieses.

Tabela 4 - Ferramenta GRADE para análise do nível de evidência.

\begin{tabular}{|c|c|c|c|c|c|c|c|c|c|c|c|c|}
\hline \multicolumn{7}{|c|}{ Avaliação de certeza } & \multicolumn{2}{|c|}{$\mathrm{N}^{0}$ de pacientes } & \multicolumn{2}{|c|}{ Efeito } & \multirow[b]{2}{*}{ Certeza } & \multirow[b]{2}{*}{ Importância } \\
\hline $\begin{array}{l}\mathrm{N}^{0} \text { de } \\
\text { estudos }\end{array}$ & $\begin{array}{l}\text { Delineamento } \\
\text { do estudo }\end{array}$ & $\begin{array}{l}\text { Risco } \\
\text { de viés }\end{array}$ & Inconsistência & $\begin{array}{c}\text { Evidência } \\
\text { indireta }\end{array}$ & Imprecisão & $\begin{array}{c}\text { Outras } \\
\text { considerações }\end{array}$ & $\begin{array}{c}\text { Exercício } \\
\text { aeróbio }\end{array}$ & Controle & $\begin{array}{l}\text { Relativo } \\
\text { (IC95) }\end{array}$ & $\begin{array}{l}\text { Absoluto } \\
\text { (IC95\%) }\end{array}$ & & \\
\hline \multicolumn{13}{|c|}{ Fração de encurtamento (avaliada com ecocardiografia e Doppler) } \\
\hline 13 & $\begin{array}{l}\text { ensaios } \\
\text { randomizados }\end{array}$ & $\begin{array}{l}\text { não } \\
\text { grave }\end{array}$ & não grave & $\begin{array}{l}\text { muito } \\
\text { grave }^{a}\end{array}$ & não grave & nenhum & 230 & 222 & - & $\begin{array}{l}\text { média } \\
5,33 \% \\
\text { maior } \\
(3,4 \text { a } \\
7,26)\end{array}$ & $\underset{\text { BAIXA }}{\oplus \oplus \bigcirc \bigcirc}$ & CRITICA \\
\hline \multicolumn{13}{|c|}{ Pressão desenvolvida no ventrículo esquerdo (avaliada com transdutor de pressão) } \\
\hline 5 & $\begin{array}{c}\text { ensaios } \\
\text { randomizados }\end{array}$ & $\begin{array}{l}\text { não } \\
\text { grave }\end{array}$ & não grave & $\begin{array}{l}\text { muito } \\
\text { grave }^{a}\end{array}$ & não grave & nenhum & 153 & 166 & - & $\begin{array}{c}\text { média } \\
24,84 \\
\text { mmHg } \\
\text { maior } \\
(20,84 \text { a } \\
28,84)\end{array}$ & $\underset{\text { BAIXA }}{\oplus \oplus \bigcirc \bigcirc}$ & CRÍTICA \\
\hline
\end{tabular}

IC: intervalo de confiança; a: estudos com animais são considerados como evidência indireta.

sobre o mesmo assunto, que mostraram que a capacidade do exercício aeróbio de prevenir e combater a cardiotoxicidade gerada pela exposição à DOX parece estar bem estabelecida em estudos com animais. ${ }^{42,43}$ No entanto, os mecanismos desse efeito ainda não foram completamente elucidados. ${ }^{44,45}$

Em uma revisão sobre os efeitos de exercício físico na resposta cardiovascular de pacientes com câncer de mama, Sturgeon et al. ${ }^{46}$ revelaram uma falta de estudos focados em cardiotoxicidade em humanos. Eles apontaram que, apesar de poucos estudos pré-clínicos indicarem uma diminuição na frequência cardíaca em repouso e na pressão arterial em pacientes que praticam exercício aeróbio durante e após a quimioterapia, esses parâmetros não são suficientes para indicar boa função cardíaca. Kirkham et al. ${ }^{47}$ no entanto, em um estudo recente de prova de conceito com pacientes com câncer de mama, encontraram resultados favoráveis na avaliação da função sistólica do grupo que praticou apenas uma sessão de exercício aeróbio de corrida na esteira de intensidade vigorosa até 24 horas antes do tratamento com DOX.

\section{Limitações}

Para ambos os desfechos, a inconsistência entre os estudos foi bastante alta, com $\mathrm{I}^{2}=87 \%(\mathrm{p}=0,00001)$ para $\Delta \mathrm{D} \%$ e $\mathrm{I}^{2}=$ $94 \%$ ( $p=0,00001$ ) para PDVE (Figuras 2 e 3). Tal inconsistência pode estar relacionada à variação ampla nos tipos de exercícios, nos protocolos de intervenção e na dosagem de DOX utilizada (Tabelas 1 e 2). Por isso, a análise de efeitos aleatórios dos resultados foi escolhida. No entanto, o resultado final obtido não parece ter sido afetado por essa grande heterogeneidade. Por exemplo, dos quatro estudos com maior peso para $\triangle \mathrm{D} \%$, dois realizaram exercício antes da DOX e dois após a DOX, os protocolos variaram de 4 a 10 semanas e a dose total de DOX variou de $3 \mathrm{mg} / \mathrm{kg}$ a $32 \mathrm{mg} / \mathrm{kg}$. Assim, não é possível concluir quais foram os melhores protocolos. 
Esses achados dos estudos pré-clínicos fornecem apenas evidência indireta em relação à prática clínica. Assim, a ferramenta GRADE teve diminuição de dois níveis no item evidência indireta, o que resultou em um baixo nível de evidência para as variáveis verificadas.

\section{Conclusão}

Esta metanálise mostrou que, em estudos com murinos expostos à DOX, o exercício aeróbio antes, durante ou depois da exposição, realizado em uma sessão única ou por até 3 meses, é uma boa estratégia para a manutenção da função do ventrículo esquerdo. Os estudos pré-clínicos mostraram que, nesse estágio da pesquisa, o exercício é uma boa estratégia não farmacológica para preservar a função cardíaca contra danos causados pela cardiotoxicidade por DOX.

\section{Contribuição dos Autores}

Concepção e desenho da pesquisa, Análise e interpretação dos dados e Revisão crítica do manuscrito quanto ao conteúdo intelectual importante: Matos MI, Rubini EC, Meireles FO,

\section{Referencias}

1. Kalil Filho R, Hajjar LA, Bacal F, Hoff PM, Diz MDP, Galas FRBG, et al. I Diretriz Brasileira de Cardio-Oncologia da Sociedade Brasileira de Cardiologia. Arq Bras Cardiol. 2011;96(2):01-52.

2. Yeh ETH, Tong AT, Lenihan DJ, Yusuf SW, Swafford J, Champion C, et al. Cardiovascular complications of cancer therapy: diagnosis, pathogenesis, and management. Circulation. 2004;109(25):3122-31.

3. Carver JR, Shapiro CL, Andrea Ng, Jacobs L, Schwartz C, Virgo KS, et al. American Society of Clinical Oncology Clinical Evidence Review on the ongoing care of adult cancer survivors: cardiac and pulmonary late effects. J Clin Oncol. 2007;25(25):3991-4008.

4. Stewart S, MacIntyre K, Hole DJ, Capewell S, McMurray JJ. More 'malignant'than cancer? Five-year survival following a first admission for heart failure. Eur J Heart Fail. 2001;3(3):315-22.

5. Minotti G, Menna P, Salvatorelli E, Cairo G, Gianni L. Anthracyclines: molecular advances and pharmacologic developments in antitumor activity and cardiotoxicity. Pharmacol Rev. 2004;56(2):185-229.

6. Herrmann J, Lerman A, Sandhu NP, Villarraga HR, Mulvagh SL, Kohli M. Evaluation and management of patients with heart disease and cancer: cardio-oncology. Mayo Clin Proc. 2014;89(9):1287-306.

7. Scott JM, Khakoo A, Mackey JR, Haykowsky MJ, Douglas PS, Jones LW. Modulation of anthracycline-induced cardiotoxicity by aerobic exercise in breast cancer. Circulation. 2011;124(5):642-50.

8. Adamsen, L, Quist M, Andersen C, Møller T, Herrstedt J, Kronborg D, et al. Effect of a multimodal high intensity exercise intervention in cancer patients undergoing chemotherapy: randomised controlled trial. BMJ. 2009 Oct;339:b3410.

9. Ashrafi J, Roshan VD. Is short-term exercise a therapeutic tool for improvement of cardioprotection against DOX-induced cardiotoxicity? An experimental controlled protocol in rats. Asian Pac J Cancer Prev. 2012;13(8):4025-30.

10. Lyon AR, Habibian M. Break a sweat to reduce cardiotoxicity-the benefits of exercise training during anthracycline chemotherapy. Eur J Prev Cardiol. 2019;26(3):301-4
Silva EB; Obtenção de dados: Matos M; Análise estatística: Silva EB; Redação do manuscrito: Matos MI, Rubini EC, Meireles FO.

\section{Potencial Conflito de Interesses}

Declaro não haver conflito de interesses pertinentes.

\section{Fontes de Financiamento}

O presente estudo não teve fontes de financiamento externas.

\section{Vinculação Acadêmica}

Não há vinculação deste estudo a programas de pósgraduação.

\section{Aprovação Ética e Consentimento Informado}

Este artigo não contém estudos com humanos ou animais realizados por nenhum dos autores.

11. Chen JJ, Wu PT, Middlekauff HR, Nguyen KL. Aerobic exercise in anthracycline-induced cardiotoxicity: a systematic review of current evidence and future directions. Am J Physiol Heart Circ Physiol. 2016;312(2):H213-22.

12. Kouzi SA, Uddin MN. Aerobic exercise training as a potential cardioprotective strategy to attenuate doxorubicin-induced cardiotoxicity. J Pharm Pharm Sci. 2016;19(3):399-410.

13. Costa MP, Castier MB, Salgado CG. Role of echocardiograms for evaluating cardiotoxicity in breast cancer treatment. Rev Bras Cardiol. 2011;24(6):382-6.

14. Campos Filho O, Zielinsky P, Ortiz J, Maciel BC, Andrade JL, Mathias Jr W, et al. Diretriz para indicações e utilização da ecocardiografia na prática clínica. Arq Bras Cardiol. 2004, 82, 11-34.

15. Cesaretti MLR, Ginoza M, Ribeiro AB, Kohlmann Jr O. Systemic hemodynamic and left ventricular function of diabetic-induced hypertensive rats. Arq Bras Endocrinol Metab. 2010;54(9):842-51.

16. Higgins J, Green S. Cochrane handbook for systematic reviews of interventions. New Jersey: John Wiley \& Sons; 2008

17. Liberati A, Altman DG, TetzlaffJ, Mulrow C, Gøtzsche PC, loannidisJPA, et al The PRISMA statement for reporting systematic reviews and meta-analyses of studies that evaluate healthcare interventions: explanation and elaboration. BMJ. 2009 July 21;339:b2700.

18. Hayward R, Lien CY, Jensen BT, Hydock DS, Schneider CM. Exercise training mitigates anthracycline induced chronic cardiotoxicity in a juvenile rat model. Pediatr Blood Cancer. 2012;59(1):149-54.

19. Hydock DS, Lien CY, Jensen BT, Parry TL, Schneider CM, Hayward R. Rehabilitative exercise in a rat model of doxorubicin cardiotoxicity. Exp Biol Med. 2012;237(12):1483-92.

20. Calvé A, Haddad R, Barama SN, Meilleur M, Sebag IA, Chalifour LE. Cardiac response to doxorubicin and dexrazoxane in intact and ovariectomized young female rats at rest and after swim training. Am J Physiol Heart Circ Physiol. 2012;302(10):H2048-57

21. Dolinsky VW, Rogan KJ, Sung MM, Zordoky BN, Haykowsky MJ, Young $\mathrm{ME}$, et al. Both aerobic exercise and resveratrol supplementation attenuate doxorubicin-induced cardiac injury in mice. Am J Physiol Endocrinol Metab. 2013;305(2):E243-53. 
22. Jensen BT, Lien CY, Hydock DS, Schneider CM, Hayward R. Exercise mitigates cardiac doxorubicin accumulation and preserves function in the rat. J Cardiovasc Pharmacol. 2013;62(3):263-9.

23. Parry TL, Hayward R. Exercise training does not affect anthracycline antitumor efficacy while attenuating cardiac dysfunction. Am J Physiol Regul Integr Comp Physiol. 2015;309(6):R675-83.

24. Lien CY, Jensen BT, Hydock, DS, Hayward R. Short-term exercise training attenuates acute doxorubicin cardiotoxicity. J Physiol Biochem. 2015;71(4):669-78.

25. Chicco JA, Schneider CM, Hayward R. Voluntary exercise protects against acute doxorubicin cardiotoxicity in the isolated perfused rat heart. Am J Physiol Regul Integr Comp Physiol. 2005;289:424-31.

26. Chicco AJ, Schneider CM, Hayward R. Exercise training attenuates acute doxorubicin-induced cardiac dysfunction. J Cardiovasc Pharmacol. 2006;47(2):182-9.

27. Hayward R, Hydock D, Gibson N, Greufe S, Bredahl E, Parry T. Tissue retention of doxorubicin and its effects on cardiac, smooth, and skeletal muscle function. J Physiol Biochem. 2013;69(2):177-87.

28. Margarida C, Duarte-Rodrigues J, Campelo M. Cardiotoxicity in anthracycline therapy: prevention strategies. Rev Port Cardiol. 2016;35(6):359-71.

29. Ascensão A, Magalhães J, Soraes J, Ferreira R, Neuparth M, Marques F, et al. Endurance training attenuates doxorubicin-induced cardiac oxidative damage in mice. Int J Cardiol. 2005;100(3):451-60.

30. Ascensão A, Magalhães J, Soraes J, Ferreira R, Neuparth M, Marques F, et al. Moderate endurance training prevents doxorubicin-induced in vivo mitochondriopathy and reduces the development of cardiac apoptosis. Am J Physiol Heart Circ Physiol. 2005;289(2):H722-31.

31. Ascensão A, Magalhães J, Soares J, Ferreira R, Neuparth M, Marques F, et al. Endurance exercise training attenuates morphological signs of cardiac muscle damage induced by doxorubicin in male mice. Basic Appl Myol. 2006;16(1):27-35.

32. Ashrafi J, Roshan VD, Mahjoub S. Cardioprotective effects of aerobic regular exercise against doxorubicin-induced oxidative stress in rat. Afr J Pharm Pharmacol. 2012;6(31):2380-8.

33. Marques-Aleixo I, Santos-Alves E, Mariani D, Rizo-Roca D, Padrão Al, RochaRodrigues $\mathrm{S}$, et al. Physical exercise prior and during treatment reduces subchronic doxorubicin-induced mitochondrial toxicity and oxidative stress. Mitochondrion. 2015 Jan;20:22-33.

34. Gewirtz DA. A critical evaluation of the mechanisms of action proposed for the antitumor effects of the anthracycline antibiotics adriamycin and daunorubicin. Biochem Pharmacol. 1999;57(7):727-41.
35. Kirkham AA, Margot KD. Exercise prevention of cardiovascular disease in breast cancer survivors. J Oncol. 2015;2015:917606.

36. Ashrafi J, Roshan VD. Pretreatment effect of running exercise on HSP70 and DOX-induced cardiotoxicity. Asian Pac J Cancer Prev. 2012;13(11):5849-55.

37. Eckel RH, Jakicic JM, Ard JD, Jesus JM, Miller NH, Hubbard VS, et al. 2013 AHA/ACC guideline on lifestyle management to reduce cardiovascular risk: a report of the American College of Cardiology/American Heart Association Task Force on Practice Guidelines. Circulation. 2014;129(25 Suppl):S76-99.

38. Perk J, De Backer G, Gohlke H, Graham I, Reiner Ž, Verschuren WM, et al. European guidelines on cardiovascular disease prevention in clinical practice (version 2012) : the fifth joint task force of the European society of cardiology and other societies on cardiovascular disease prevention in clinical practice (constituted by representatives of nine societies and by invited experts). Int J Behav Med. 2012;19(4):403-88.

39. Leaf DA, Kleinman MT, Hamilton M, Deitrick RW. The exercise-induced oxidative stress paradox: the effects of physical exercise training. Am J Med Sci. 1999;317(5):295-300.

40. Naci H, loannidis JP. Comparative effectiveness of exercise and drug interventions on mortality outcomes: metaepidemiological study. BMJ. 2013 Oct 01;347:f5577.

41. Puetz TW, Beasman KM, O'Connor PJ. The effect of cardiac rehabilitation exercise programs on feelings of energy and fatigue: a meta-analysis of research from 1945 to 2005. Eur J Cardiovasc Prev Rehabil. 2006;13(6):886-93.

42. Hahn VS, Lenihan DJ, Ky B. Cancer therapy-induced cardiotoxicity: basic mechanisms and potential cardioprotective therapies. J Am Heart Assoc. 2014;3(2):e000665.

43. Wonders KY, Reigle BS. Trastuzumab and doxorubicin-related cardiotoxicity and the cardioprotective role of exercise. Integr Cancer Ther. 2009;8(1):17-21.

44. Chen B, Peng X, Pentassuglia L, Lim CC, Sawyer DB. Molecular and cellular mechanisms of anthracycline cardiotoxicity. Cardiovasc Toxicol. 2007;7(2):114-21.

45. Maia TN, Araujo GBRD, Teixeira JAC, Alves Jr ED, Dias KP. Cardiotoxicity of doxorubicin treatment and physical activity: a systematic review. Int J Cardiovasc Sci. 2017;30(1):70-80.

46. Sturgeon KM, Ky B, Libonati JR, Schmitz KH. The effects of exercise on cardiovascular outcomes before, during, and after treatment for breast cancer. Breast Cancer Res Treat. 2014;143(2):219-26.

47. Kirkham AA, Shave RE, Bland KA, Bovard JM, Eves ND, Gelmon KA, et al. Protective effects of acute exercise prior to doxorubicin on cardiac function of breast cancer patients: a proof-of-concept RCT. Int J Cardiol. $2017 ; 245: 263-70$. 